BIOCYT Biología, Ciencia y Tecnología, 10(37): 656-671, 2017 (enero-marzo)

ISSN 2007-2082

www.iztacala.unam.mx/biocyt

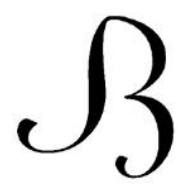

Publicada en la Facultad de Estudios Superiores Iztacala, Universidad Nacional Autónoma de México

\title{
ESTRUCTURA DE TALLAS DE MACROBRACHIUM TENELLUM (DECAPODA: PALAEMONIDAE) EN EL ESTERO EL SALADO, PUERTO VALLARTA, JALISCO, MÉXICO
}

\author{
SIZE STRUCTURE OF MACROBRACHIUM TENELLUM \\ (DECAPODA: PALAEMONIDAE) IN THE ESTUARY EL SALADO, \\ PUERTO VALLARTA, JALISCO, MEXICO
}

\author{
Erick Emmanuel Jáuregui-Velázquez* y Sandra Quetzalli Bárcenas-Gutiérrez \\ Módulo de Manejo de Recursos Naturales, Facultad de Estudios Superiores Iztacala, Universidad Nacional \\ Autónoma de México. Av. De los Barrios, No. 1, Los Reyes Iztacala, Tlalnepantla, Estado de México, México. \\ C.P. 54090. * javenoctiluca@hotmail.com, quetzy91@hotmail.com
}

\begin{abstract}
The prawns of the family Palaemonidae Rafinesque, 1815 are the most diverse group of order Decapoda; shown a wide geographic and batimetric distributions, several species are considerate with economic value. In Mexico have information gaps regarding to Macrobrachium tenellum Smith, 1871 population, the influence of seasonality and abiotic factors as temperature and salinity, besides another ecological processes. Five samplings were made between August 2013 and July 2014; May 2014 was the most abundant month with 246 individuals recorded; meanwhile, February 2014 was the month with lesser individuals recorded. Post-larvae stages were the most numerous throughout of the year, and the mature adults were most scarce. In estuary El Salado, organisms of M. tenellum did not reach commercial sizes, males group reached greater total length and weight in average. It was observed that temperature and fluvial precipitation have an effect on the number of organisms. Keywords: abundance, growth, Macrobrachium tenellum, prawns, sex ratio, size, weight.
\end{abstract}

Manuscrito recibido el 10 de agosto de 2016 aceptado el 25 de noviembre de 2016. 


\section{RESUMEN}

Los langostinos de la familia Palaemonidae Rafinesque ,1815 son el grupo más diverso del orden de los decápodos; muestran una amplia distribución geográfica y batimétrica, varias especies son consideradas de valor económico. En México existen vacíos de información respecto a la población de $M$. tenellum Smith, 1871, la influencia de la estacionalidad y factores abióticos como la temperatura y salinidad, además de otros procesos ecológicos. Cinco muestreos fueron realizados entre agosto de 2013 y julio de 2014; mayo de 2014 fue el mes más abundante con 246 individuos registrados; mientras que febrero de 2014 fue el mes con menos individuos registrados. Etapas postlarvales fueron las más numerosas durante todo el año, los adultos maduros fueron los más escasos. En el estero El Salado, individuos de M. tenellum no alcanzaron tallas comerciales, los machos alcanzaron mayor longitud total y peso promedio. Se encontró que el número de organismos está influenciado por la temperatura y la precipitación pluvial.

Palabras clave: abundancia, crecimiento, Macrobrachium tenellum, langostino, proporción sexual, tallas, peso.

\section{INTRODUCCIÓN}

Dentro de los invertebrados, la clase de los crustáceos tiene gran importancia desde el punto de vista biológico y económico. Los decápodos dentro de los cuales se incluyen camarones, cangrejos, jaibas, langostinos y langostas (Brusca y Brusca, 2003); son necesarios alrededor del mundo para la satisfacción de las necesidades de alimentación del hombre, ya sea como producto alimenticio directo de la pesca o como recurso fundamental en las cadenas tróficas de otros recursos pesqueros (Espinosa-Chaurand et al., 2011).

Los langostinos de la familia Palemonidae son los más diversos dentro del orden Decapoda, su distribución geográfica y batimétrica es amplia y están representados un gran número de especies en los sistemas marinos, estuarinos y dulceacuícolas (Hernández-Sandoval, 2008). De las 48 especies registradas en el Pacífico Centro-Oriental, los palemónidos ocupan el segundo lugar en abundancia entre las familias del infraorden Caridea (Espinosa-Chaurand et al., 2011).

El género Macrobrachium Bate, 1868, ha sido de gran interés biológico debido al número de especies que lo conforman, su distribución geográfica y su importancia económica en muchos países (Vega-Villasante et al., 2011b), constituye uno de los géneros más abundantes y ampliamente distribuidos de crustáceos con cerca de 200 especies descritas (Hernández-Sandoval, 2008), son abundantes en toda la franja tropical y subtropical de Asia (mayor diversidad )y América donde también existe un importante número de especies, algunas endémicas (Murphy y Austin, 2005).

En México se tienen registradas 17 especies del género Macrobrachium: Macrobrachium occidentale Holthuis, 1950, Macrobrachium americanum Bate, 1968, Macrobrachium michoacanus Villalobos y Nates, 1990, Macrobachium tenellum Smith, 1871 y Macrobrachium digueti Bouvier, 1895 habitan cuerpos dulceacuícolas cercanos a las costas del Pacífico, mientras que Macrobrachium acanthurus Wiegmann, 1836, Macrobrachium acherontium Holthuis, 1977, Macrobrachium carcinus Linnaeus, 1758, Macrobrachium heterochirus Wiegmann, 1836, Macrobrachium nattereri Heller, 1862, Macobrachium vicconi Roman, Ortega y Mejía, 2000, Macrobrachium totonacum Mejía, Álvarez y Hartnoll, 2003, Macrobrachium quelchi De Man, 1900 y Macrobrachium tuxtlaense Villalobos y Álvarez, 1999, se encuentran del lado del Atlántico, Macrobrachium rosembergii De Man, 1879 es una especie exótica introducida a México con fines de cultivo en el estado de Guerrero en 1978 (Hernández-Sandoval, 2008), Macrobrachium hobbsi Villalobos y Rodríguez, 1990 y Macrobrachium olfersii Wiegmann, 1836 se encuentran en el océano Pacifico y el Atlántico (Hernández et al., 2007; Villalobos-Hiriart et al.,1993). 
En México, existen vacíos sobre diferentes aspectos de la biología básica de M. tenellum, respecto a la dinámica poblacional y los factores abióticos que afectan a la especie. La mayoría de la literatura existente se ha enfocado al cultivo de algunas especies del género, como M. carcinus y Macrobrachium amazonicum Heller, 1862 (García-Guerrero et al., 2013) consideradas de importancia económica. Debido a lo anterior el objetivo de este trabajo fue relacionar la estructura de tallas de M. tenellum y la influencia de algunos parámetros ambientales.

\section{MATERIALES Y MÉTODOS}

Área de estudio

El Salado es un estero urbano ubicado en el estado de Jalisco, en el municipio de Puerto Vallarta; se localiza a $20^{\circ} 39^{\prime} 21^{\prime \prime}$ de latitud Norte y a $13^{\prime} 34^{\prime \prime}$ de longitud Oeste, sobre la planicie costera del Pacífico Mexicano, en el punto de unión entre dos zonas de contacto: la Sierra Madre Occidental y la Sierra Madre del Sur, se encuentra en la región Ameca, que se localiza en la zona centro noroeste del estado de Jalisco; ocupa una extensión de 180 hectáreas (1.8 km²) (Fig. 1).

Se realizaron cinco capturas entre agosto de 2013 y julio de 2014 en cinco puntos del estero El Salado, cercanos a la desembocadura de ríos (Fig. 2). Las recolectas se hicieron empleando una red de cuchara de $70 \mathrm{~cm}$ de diámetro y luz de malla de $5 \mathrm{~mm}$.

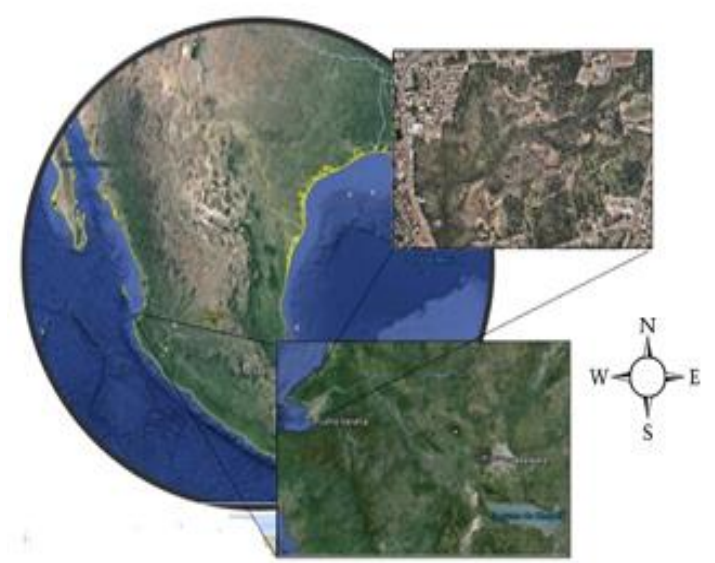

Fig. 1. Área de estudio.

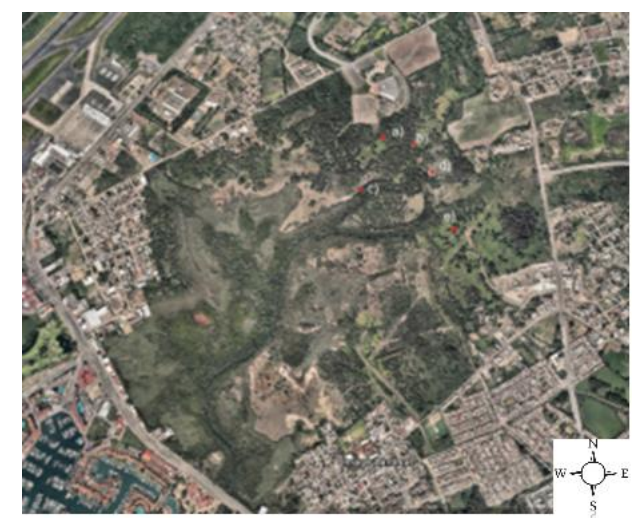

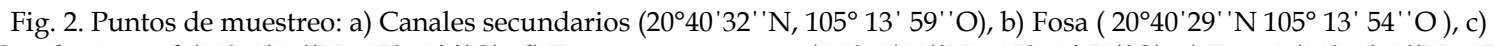
Canal principal $\left(20^{\circ} 40^{\prime} 24^{\prime \prime} \mathrm{N} 105^{\circ} 13^{\prime}\right.$ ' 'O), d) Estanques pequeños ( $20^{\circ} 40^{\prime} 23^{\prime \prime} \mathrm{N}, 105^{\circ} 13^{\prime} 52^{\prime}$ ' O), e) Fosa $2\left(20^{\circ} 40^{\prime} 12^{\prime}\right.$ ' $\mathrm{N} 105^{\circ}$ 13 ' 54 ' 'O). 
Los organismos fueron identificados siguiendo las claves de Holtuis (1952) y Hendrickx (1995) y criterios de Espinosa et al., (2011). El sexo se determinó empleando las claves y criterios de Román-Contreras (1979). Se estimó la proporción sexual para cada mes. Todos los organismos fueron medidos en longitud total (del rostro hasta la parte anterior del telson). Se registró el peso en gramos y se relacionaron peso y talla mediante la ecuación: $\mathrm{W}=\mathrm{a} \mathrm{L}^{\mathrm{b}}$, (Ricker, 1975), donde $\mathrm{W}=\mathrm{Peso}$, $\mathrm{L}=$ longitud, $\mathrm{a}=$ intercepto $\mathrm{y} \mathrm{b}=$ ordenada al origen, posteriormente se calcularon $\mathrm{a}$ y $\mathrm{b}$ a partir de una transformación logarítmica (Zar, 2009): $\log W=\operatorname{Loga}+b \log L$, donde $b$ es el coeficiente de crecimiento. Los valores para determinar alometría fueron $(b<3 \quad o \quad b>3)$ o isometría $(b=3)$ (Olugbenga y Taiwo, 2013). El factor de condición fisiológica $(\mathrm{Kn})$ fue calculado mensualmente para machos y hembras empleando la ecuación $\mathrm{K}=100^{*} \mathrm{~W}^{*} \mathrm{~L}^{-3}$ (LeCren, 1951, estableció que el factor de condición $\mathrm{K}$ es determinado de la relación entre largo de caparazón y peso corporal) donde, W= peso corporal (g) y L= largo del caparazón (Pauly, 1984; Olugbenga y Taiwo, 2013). Para este caso se usó el peso total $(\mathrm{Pt})$ y la longitud total $(\mathrm{Lt})$.

Se analizaron las posibles diferencias entre el número de organismos de cada mes, el análisis se realizó con el software estatistica 7.0 de statsoft. Se usó una base de datos codificada por meses y estadios. Se comprobó que los datos no presentan normalidad por medio de las pruebas de Shapiro-Wilks y liliefors (en estas pruebas la hipótesis nula refiere que las muestras fueron tomadas de una población con distribución normal). Se aplicó la prueba de Levene para conocer si los datos eran homocedasticos, y la prueba de Kruskall Wallis para medir la varianza de las medianas por mes. Las mismas pruebas se aplicaron para probar la normalidad de los datos de tallas y pesos por mes. Se aplicó la prueba de U de Mann Whitney. Estas pruebas se aplicaron para conocer si existen diferencias significativas en el tamaño y peso de los organismos en cada mes. La prueba se calcula sobre la base de las sumas de rango en lugar de las medias. La prueba de U de Mann Whitney (Z) son significativas solo si $\mathrm{P}<0.05$, bajo la hipótesis alternativa, los valores de una de las muestras tienden a exceder a los de la otra.

Durante las capturas se registraron la temperatura (grados centígrados) y salinidad (expresada en unidades prácticas de salinidad) (UPS) con un multiparamétrico (Oakton 120 de 0 a 50 UPS). Los valores de salinidad se analizaron mediante una matriz de correlaciones mediante el software Statsoft 2007 (en esta, el valor de R cuantifica el efecto que se genera entre las variables X) para observar el comportamiento de la misma en los meses de captura.

\section{RESULTADOS}

Salinidad

A lo largo del muestreo se registró un intervalo entre 0 y 7.2 UPS. En agosto se registró un intervalo de 0 a 5, con promedio de 2.4 UPS, en octubre fue de 1 a 6.8 con promedio de 3.1 UPS, en febrero se registró un intervalo entre 0 y 7 con un promedio de 3.8 UPS, en mayo se observó un intervalo entre 3 y 7.2 UPS con un promedio de 4.9, en julio la salinidad registrada estuvo entre 2 y 6 con un promedio de 3.3 UPS (Fig.3).

\section{Temperatura}

La temperatura del agua se mantuvo en un intervalo de 23 a $28.7^{\circ} \mathrm{C}$. Febrero fue el mes menos caluroso con $23^{\circ} \mathrm{C}$ (Fig.3). 
Precipitación

La precipitación fue de $136.5 \mathrm{~mm}$ en agosto, en octubre fue $108.2 \mathrm{~mm}$, en febrero $0 \mathrm{~mm}$, mayo $7.3 \mathrm{~mm}$ y julio $206.8 \mathrm{~mm}$.

El análisis de matriz de correlaciones entre las variables ambientales y la abundancia mensual arrojó que la relación de la temperatura y la abundancia es directamente proporcional en un $41 \%$, asimismo la precipitación mostró una relación directamente proporcional en $45 \%$ con la temperatura, esto con $\mathrm{p}<0.05000$ en ambos casos (Fig.4).

Abundancia

Se capturaron 633 organismos en total, 224 machos (35.39\%) y 409 hembras (64.61\%). En agosto se colectaron 166 organismos, en octubre 89 organismos, en febrero se colectaron 12 organismos, en mayo 246 y en julio se capturaron 120 organismos (Fig. 5). Para las abundancias agrupadas por mes, la prueba de Kruskall Wallis mostró una diferencia significativa en las medianas. $\mathrm{H}(\mathrm{Gl}=4, \mathrm{~N}=25)=16.5 \mathrm{Y} \mathrm{P}=0.023(<.05)$, con $\mathrm{X}^{2}{ }_{\text {tablas }}$ critica=9.48 por lo que se observó que existen diferencias significativas en las medianas de la abundancia mensual.

Tallas

Los organismos más abundantes fueron los de tallas entre 1.2 y $2.4 \mathrm{~cm}$ (313 individuos), solo se observaron 40 organismos maduros (Fig. 6). La talla mínima en machos fue $1.3 \mathrm{~cm}$ y la máxima $8.7 \mathrm{~cm}$ con un promedio de $3.07 \pm 1.35 \mathrm{~cm}$. La talla máxima se presentó en julio $(8.7 \mathrm{~cm})$. La talla mínima en hembras fue $1.2 \mathrm{~cm}$ y la máxima 7.2 , con un promedio de $2.7 \pm 1.08 \mathrm{~cm}$. La talla máxima se presentó en mayo $(7.2 \mathrm{~cm})$ (Fig.7).

Las pruebas de $\mathrm{U}$ de Mann Whitney para longitud total entre machos y hembras en los meses de agosto $(Z=-2.71$ y $p=0.006)$, febrero $(Z=-2.7 y p=0.0057)$ y mayo $(Z=-2.76 \quad$ y $p$ $=0.0057)$ mostraron que los valores de una de las muestras exceden a los de la otra. Para los meses de octubre y julio la prueba no mostró diferencias entre longitudes.

Peso

En agosto se registró un intervalo de peso para machos de 0.04 a $0.16 \mathrm{~g}$ (promedio de $0.078 \pm$ $0.029 \mathrm{~g}$ ), en hembras fue de $0.02 \mathrm{a} 0.14 \mathrm{~g}$ (promedio de $0.062 \pm 0.023 \mathrm{~g}$ ). En octubre se registró un intervalo de peso para machos de 0.04 a $3.68 \mathrm{~g}$ (promedio de $0.204 \pm 0.28 \mathrm{~g}$ ), en hembras fue de 0.03 a $1.43 \mathrm{~g}$ (promedio de $0.107 \pm 0.181 \mathrm{~g}$ ). En febrero se registró un intervalo de peso para machos de 0.15 a $0.39 \mathrm{~g}$ (promedio de $0.24 \pm 0.103 \mathrm{~g}$ ), en hembras fue de 0.11 a 0.54 (promedio $0.31 \pm 0.18 \mathrm{~g}$ ). En mayo se registró un intervalo de peso para machos de 0.09 a $2.54 \mathrm{~g}$ (promedio de $0.653 \pm 0.604 \mathrm{~g}$ ), en hembras fue de 0.13 a $3.66 \mathrm{~g}$ (promedio de $0.46 \pm 0.54 \mathrm{~g}$ ). En julio se registró un intervalo de peso para machos de 0.06 a $6.13 \mathrm{~g}$ (promedio de $0.680 \pm 1.14 \mathrm{~g}$ ), en hembras fue de 0.04 a $2.0 \mathrm{~g}$ (promedio de $0.37 \pm 0.36 \mathrm{~g}$ (Fig. 7).

Las pruebas de $\mathrm{U}$ de Mann Whitney para los pesos entre machos y hembras en los meses de agosto $(Z=-3.1, P=0.0019)$ y mayo $(Z=-2.81$ y $p=0.0048)$ mostraron que los valores de una de las muestras exceden a los de la otra. Para los otros meses la prueba no mostró diferencias entre pesos. La relación entre peso-talla en la población total se ajustó a un modelo potencial $\mathrm{W}=0.014 \mathrm{~L}^{2.621}$ con un coeficiente de determinación $\left(\mathrm{r}^{2}=0.895\right)$ (la relación observada es alométrica negativa $\left.b<3\right)$. En machos la relación se ajustó al modelo $\mathrm{W}=0.0135 \mathrm{x}^{2.6523}$ con un coeficiente de determinación $\mathrm{r}^{2}=$ 0.8907, en hembras fue $\mathrm{W}=0.0144 \mathrm{x}^{2.59}$ con un coeficiente de determinación $\mathrm{r}^{2}=0.8903$ (Fig. 8). 
Macrobrachium tenellum mostró alometría negativa $(b<3)$, esto se establece según la pendiente de las regresiones de relación peso y talla, en hembras la pendiente $b=2.5 \mathrm{y}$ en machos $b=2.6$. El crecimiento es isométrico cuando $b=3$. Cuando $b>3$, lo que indica que los individuos han incrementado su peso en mayor proporción que su longitud, presentando un crecimiento alométrico positivo. Para este caso cuando $b<3$, los individuos incrementan en longitud relativa más que en peso.

Se observó un intervalo de 0.5 a $6.50 \mathrm{gcm}^{-3}$ en el factor de condición (K) para las hembras (promedio $1.07 \pm 0.6 \mathrm{gcm}^{3}$ ), en machos se observó un intervalo de 0.1 a $4.1 \mathrm{gcm}^{-3}$ (promedio $0.99 \pm$ $0.4 \mathrm{gcm}^{-3}$ ) (Tabla 1, Fig. 9). El factor K varió en el mes de febrero, en hembras fue $2.9 \pm 2.45$ y en machos $0.5 \pm 0.4$.

Estructura de tallas

En agosto la población estuvo compuesta por organismos indiferenciados morfológicamente (menos de $1 \mathrm{~cm}$ de Lt) y 166 organismos en estadio postlarval (entre 1.2 y $2.4 \mathrm{~cm}$ de Lt). En octubre la población se compuso de 78 organismos en estadio postlarval (entre 1.2 y 2.4 $\mathrm{cm}$ de Lt), nueve organismos en estadio juvenil (entre 2.5 y $3.5 \mathrm{~cm}$ de Lt) y dos organismos en estadio adulto (entre 3.6 y $5 \mathrm{~cm}$ ). En febrero la población estuvo compuesta de cinco organismos postlarvales (entre 1.2 y $2.4 \mathrm{~cm}$ de Lt), seis organismos juveniles (entre 2.5 y $3.5 \mathrm{~cm} \mathrm{de} \mathrm{Lt)} \mathrm{y} \mathrm{un}$ organismo en etapa madura con una longitud total de $5.3 \mathrm{~cm}$. En mayo la población estuvo compuesta de 48 organismos en etapa postlarval (entre 1.2 y $2.4 \mathrm{~cm}$ de Lt), 109 organismos en etapa juvenil (entre 2.5 y $3.5 \mathrm{~cm}$ de Lt), 61 organismos en etapa adulta (entre 3.6 y $5.0 \mathrm{~cm} \mathrm{de} \mathrm{Lt)} \mathrm{y} 28$ organismos en etapa madura (más de $5.0 \mathrm{~cm}$ de Lt). En julio la población estuvo compuesta de 16 organismos en estadio postlarval (entre 1.2 y $2.4 \mathrm{~cm}$ de Lt.), 53 organismos en estadio juvenil (entre 2.5 y $3.5 \mathrm{~cm}$ de Lt), 39 organismos en etapa adulta (entre 3.6 y $5.0 \mathrm{~cm}$ de Lt) y 12 organismos en etapa madura (más de $5.0 \mathrm{~cm}$ de Lt) (Tabla 2).

\section{Proporción sexual}

La proporción sexual favoreció a las hembras en todos los estadios y en todos los muestreos (M:H=1:1.82) (Fig.10), en los que el porcentaje de machos resultó menor al $45 \%$ de la población (Tabla3).

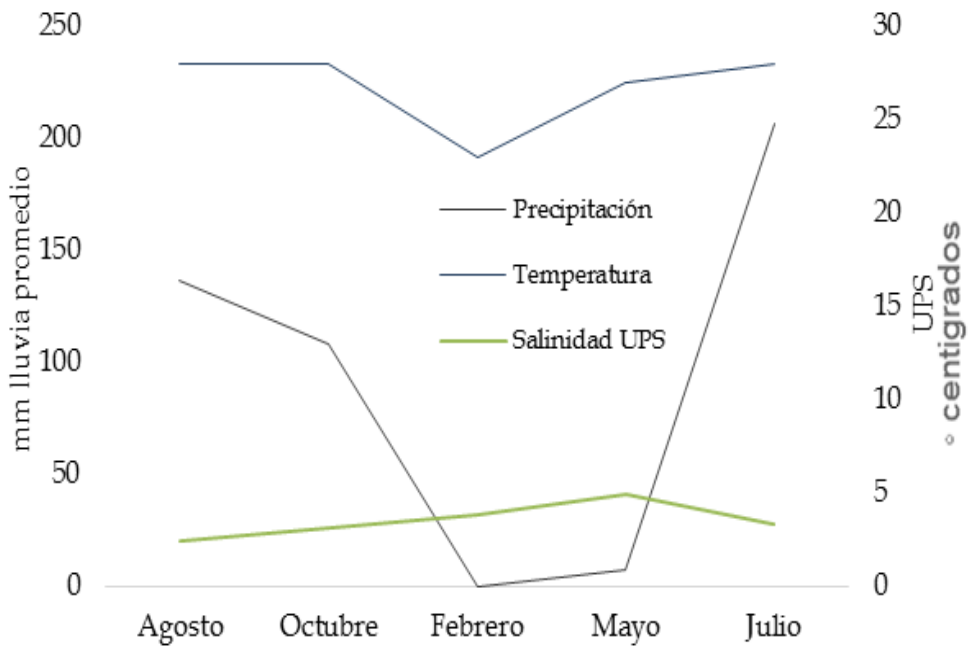

Fig.3. Temperatura, salinidad y precipitación para cada muestreo. 


\begin{tabular}{|c|c|c|c|c|}
\hline \multirow[b]{2}{*}{ Variable } & \multicolumn{4}{|c|}{$\begin{array}{l}\text { Correlations (regresion) } \\
\text { Marked correlations are significant at } p<, 05000 \\
\mathrm{~N}=25 \text { (Casewise deletion of missing data) }\end{array}$} \\
\hline & precipitacion & abundancia & salinidad & temperatura \\
\hline precipitacion & 1,000000 & 0,052142 & $-0,184706$ & 0,455179 \\
\hline abundancia & 0,052142 & 1,000000 & 0,301773 & 0,414972 \\
\hline salinidad & $-0,184706$ & 0,301773 & 1,000000 & 0,332739 \\
\hline temperatura & 0,455179 & 0,414972 & 0,332739 & 1,000000 \\
\hline
\end{tabular}

Fig. 4. Matriz de correlación de variables precipitación, salinidad, temperatura y abundancia, resultado del software Statistica 7.0

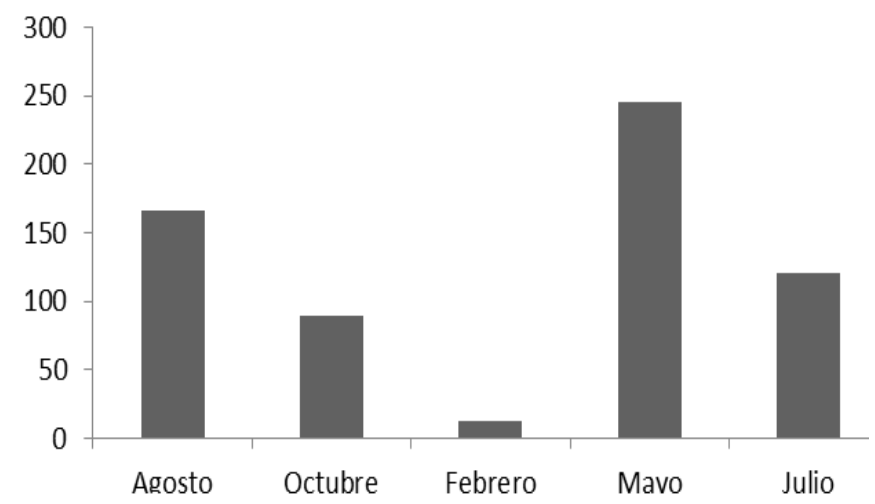

Fig.5. Abundancia mensual de M. tenellum.

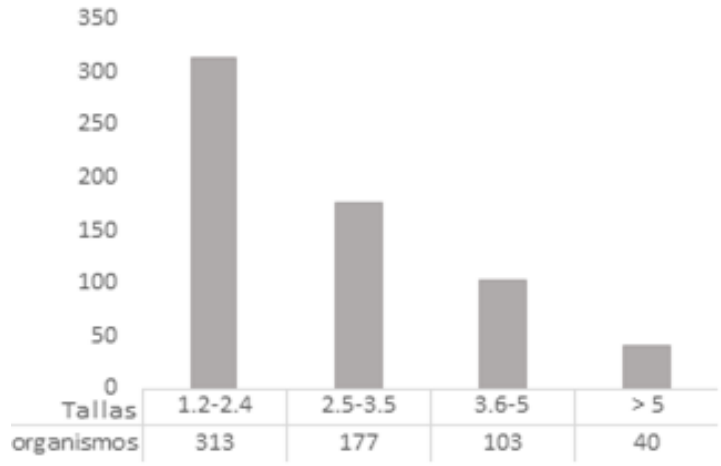

Fig. 6. Frecuencias de tallas de M. tenellum en el estero El salado. 


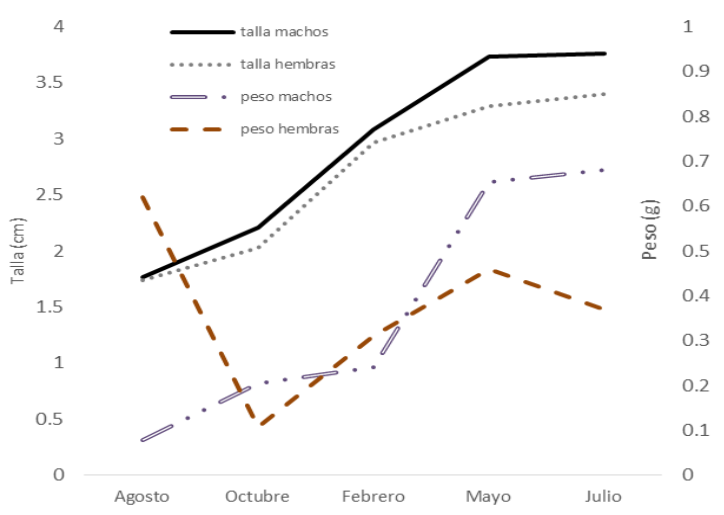

Fig. 7. Talla y peso promedio mensuales por sexo entre agosto de 2013 y julio de 2014.
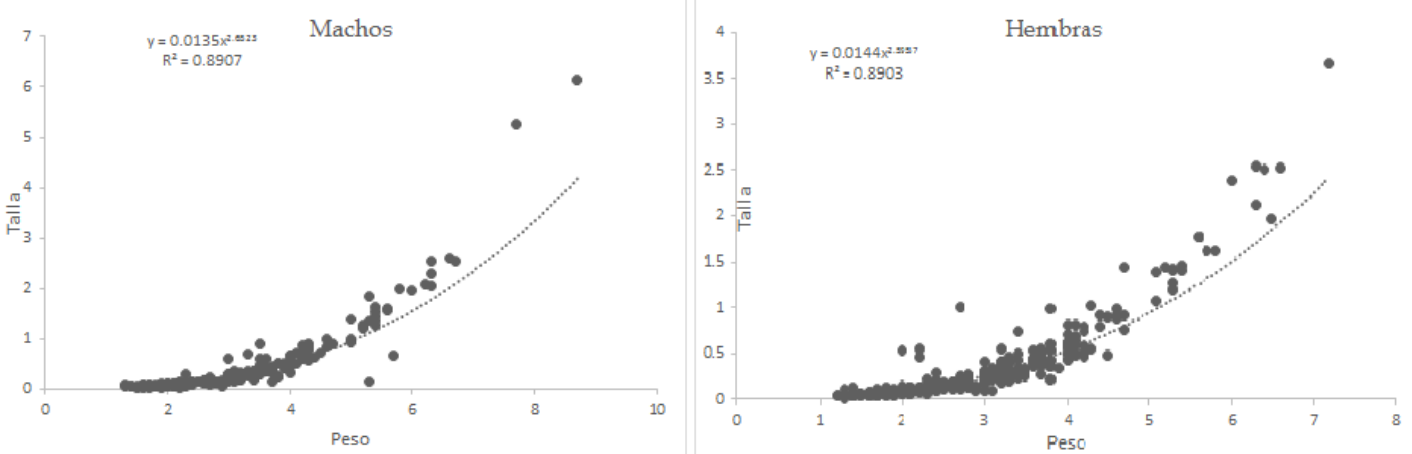

Fig.8. Relación peso-talla de la población de M. tenellum. La ecuación que describe la relación peso-talla en machos fue $\mathrm{W}=0.0135 \mathrm{x}^{2.6523}$ con un coeficiente de determinación $R^{2}=0.8907$, en hembras fue $w=0.0144 x^{2.59}$ con un $R^{2}=0.8903$ y para la poblacion total $W=0.014 \mathrm{~L}^{2.621}$ con un $\mathrm{R}^{2}=0.895$ ( $\mathrm{b}$ es el tipo de crecimiento). 
Tabla 1. Sumario de Factor de condición promedio e intervalos para cada sexo.

\begin{tabular}{lllllll}
\multicolumn{5}{c}{ promedios por mes y sexo } & & \multicolumn{2}{c}{ intervalo } & $\mathrm{g} / \mathrm{cm}^{3}$ \\
mes & K hembras & K machos & promedio & $\pm \mathrm{SD}$ & mínimo & máximo \\
\hline agosto & 1.205 & 1.239 & 1.2 & 0.54 & 0.4 & 5 \\
octubre & 1.07 & 1.04 & 1.1 & 0.265 & 0.4 & 1.8 \\
febrero & 2.9 & 0.53 & 1.9 & 2.15 & 0.1 & 6.5 \\
mayo & 1.019 & 1.04 & 1 & 0.462 & 0.3 & 4.7 \\
julio & 0.74 & 0.8 & 0.7 & 0.16 & 0.3 & 1.068
\end{tabular}

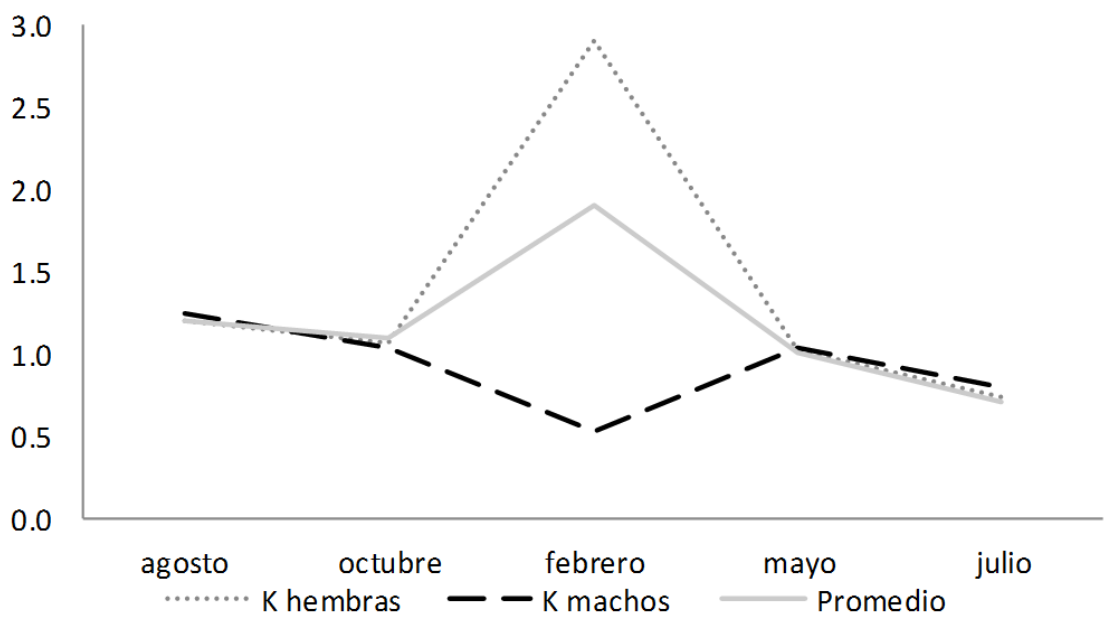

Fig. 9. Tendencia del factor de condición total y por sexos.

Tabla 2. Estructura por tallas en los meses de agosto y octubre (2013), febrero, mayo y julio (2014).

\begin{tabular}{ccccccc} 
estadio & tallas & agosto & octubre & febrero & mayo & julio \\
\hline postlarval & $1.2-2.4$ & 166 & 78 & 5 & 48 & 16 \\
juvenil & $2.5-3.5$ & 0 & 9 & 6 & 109 & 53 \\
adulto & $3.6-5$ & 0 & 2 & 1 & 61 & 39 \\
adulto maduro & $\leq 5.1$ & 0 & 0 & 0 & 28 & 12 \\
Total & & 166 & 89 & 12 & 246 & 120
\end{tabular}

Tabla 3. Proporción sexual de M. tenellum en cada muestreo. 
BIOCYT, 10(37): 656-671, 2017, enero-marzo. Erick Emmanuel Jáuregui-Velázquez. Estructura de tallas de Macrobrachium.

\begin{tabular}{cccccc} 
mes de colecta & abundancia & $\begin{array}{c}\text { hembras (\# de } \\
\text { organismos) }\end{array}$ & $\begin{array}{c}\text { porcentaje } \\
\%\end{array}$ & $\begin{array}{c}\text { machos (\# de } \\
\text { organismos) }\end{array}$ & $\begin{array}{c}\text { porcentaje } \\
\%\end{array}$ \\
\hline agosto & 166 & 111 & 66.867 & 55 & 33.13 \\
octubre & 89 & 57 & 64.04 & 32 & 35.95 \\
febrero & 12 & 7 & 58.33 & 5 & 41.66 \\
mayo & 246 & 167 & 67.886 & 79 & 32.1138 \\
julio & 120 & 67 & 55.83 & 53 & 44.16 \\
Total & 633 & 409 & $64.61 \%$ & 224 & $35.53 \%$
\end{tabular}

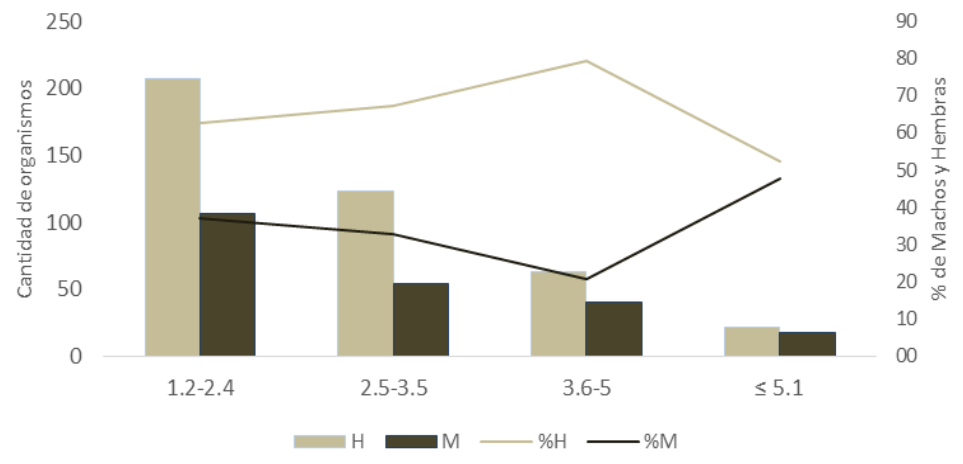

Fig. 10. Estructura tallas por sexo.

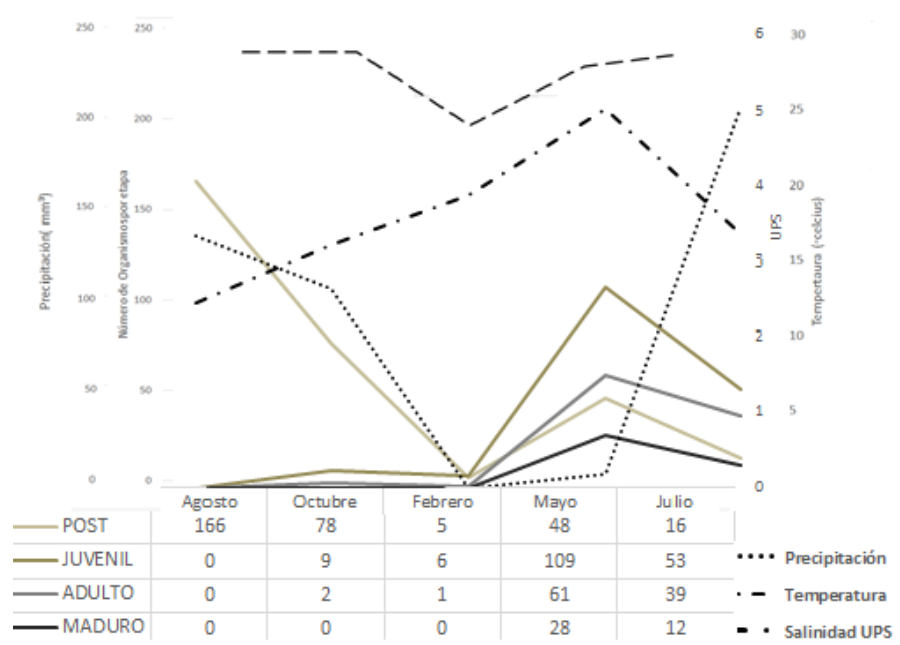

Fig. 11. Abundancia de clases de M. tenellum, temperatura, salinidad y precipitación (agosto 2013-julio 2014). 


\section{DISCUSIÓN}

El análisis de correlación mostró una ligera relación proporcional entre abundancia y temperatura, la salinidad parece no tener influencia en la abundancia mensual, esto debido a que la salinidad se mantuvo baja a lo largo del año (Fig. 3). La zona de recolecta es influenciada por un río que se encuentra cerca, lo que posiblemente afecta la salinidad. El nivel del agua depende de las lluvias y de ellas la dilución de sal y otros minerales (Cifuentes et al., 1997). Espinosa y Rodríguez (1986) mencionan que los estadios, postlarvales y juveniles del genero Macrobrachium prefieren salinidades entre 7 y 22 UPS; Vega-Villasante et al. (2011a) observaron que M. tenellum tiene la capacidad de tolerar un intervalo salino de 0 a 35 UPS, los mismos autores mencionan que las larvas prefieren una salinidad entre 5 y 15 UPS, mientras que los juveniles, adultos y organismos maduros prefieren aguas dulces. Por lo que la salinidad presente en el estero El Salado propicia la presencia de diferentes estadios al mismo tiempo en los mismos espacios. Cabe resaltar que los estadios postlarvales fueron más numerosos en puntos ligeramente salados (canal principal y estanques pequeños, 6 y 7.2 UPS respectivamente), siendo el canal principal donde se capturó un mayor número de postlarvas.

Algunos estudios se han abocado a establecer el efecto de factores ambientales con particular interés en la temperatura; Flores et al. (2012), determinaron bajo condiciones de laboratorio que $M$. tenellum prefiere temperaturas entre 30 y $33^{\circ} \mathrm{C}$ (con un óptimo de $32^{\circ} \mathrm{C}$ ). $\mathrm{Al}$ respecto autores como Ponce-Palafox et al. (2002 y 2006) mencionan que éste intervalo de temperatura es adecuado para el cultivo de varias especies del género Macrobrachium; sin embargo, esto no se ha implementado en las diferentes regiones en las que esta especie se considera nativa.

Las temperaturas más altas y los meses más lluviosos correspondieron a los meses con mayor abundancia (agosto de 2013, mayo y julio de 2014) (Fig. 3). Con los registros de precipitación se puede observar una marcada época de lluvias que para 2013, inició en julio y terminó la primer semana de enero de 2014; la época de secas inició a mediados de enero y duró hasta inicios de mayo de 2014, la época de lluvias comenzó de nuevo a mediados de mayo 2014 y se extendió hasta diciembre del mismo año (Fig. 3) (SCT SENEAM, 2015).

De acuerdo al análisis de matriz de correlación (Fig. 4.), la temperatura y la precipitación están relacionadas directamente, situación que contrasta en las colectas de mayo y febrero (Fig. 11). Román-Contreras (1979) menciona que existe relación entre la temperatura registrada en los puntos de recolecta y el número de organismos capturados, así mismo menciona que en la laguna de Tres Palos, Guerrero, México, se observaron dos picos de abundancia, de enero a junio (correspondiente con una parte de secas), y de agosto a octubre (mitad de la estación lluviosa), en las que se capturaron 200 ejemplares.

En la laguna de Coyuca, Guerrero, México, en agosto se reportaron organismos de Lt de 11 $\mathrm{cm}$ (promedio de $5.5 \mathrm{~cm}$ y moda 6.5). En esta laguna los organismos de tallas más pequeñas se encontraron entre diciembre y marzo (Román-Contreras, 1991). Signoret y Brailovsky (2000) reportaron para el mismo lugar intervalos de tallas entre 1.6 y $13.9 \mathrm{~cm}$ de Lt, las frecuencias mayores de talla estuvieron entre 6.0 y $9.0 \mathrm{~cm}$ de Lt. En el área de estudio las tallas menores a 2.5 $\mathrm{cm}$ de Lt fueron abundantes en agosto, disminuyendo hacia febrero, aunque estas tallas pequeñas se pueden encontrar a lo largo del año, esto coincide con los valores de temperatura, disminución de la precipitación en octubre y un ligero aumento de salinidad (Fig. 11). Espinosa-Chaurand et al. (2011), mencionan que las larvas se desarrollan muy cerca del mar, realizan la metamorfosis y al pasar a estadio de postlarvas prefieren condiciones de salinidad y temperatura diferentes a las de un ambiente costero, por lo que comienzan a migrar a las partes altas. Bauer (2011a) establece que 
los organismos del género Macrobrachium, realizan migraciones río arriba a medida que avanza su desarrollo. En El Salado la precipitación fue mayor en los meses de junio, julio y agosto de 2013, lo que pudo haber favorecido la presencia de organismos indiferenciados (500) y en etapas postlarval y juvenil en febrero, mes de la primera colecta (Fig. 11). Esto puede ocurrir puesto que en junio los adultos pueden aprovechar las primeras lluvias para llegar a las zonas de reproducción. Los organismos de tallas mayores a $3.5 \mathrm{~cm}$ (adultos y maduros) fueron numerosos en mayo y julio de 2014 pero también se observaron postlarvas y juveniles $(<3.6 \mathrm{~mm})$; esto coincide con la precipitación y la temperatura elevada.

En mayo y julio se observaron machos con una longitud máxima de $8.7 \mathrm{~cm}$. y un peso máximo de $6.13 \mathrm{~g}$. Las tallas y pesos registrados en el presente estudio (Fig. 7) difieren de lo reportado para langostinos de la misma especie en otros cuerpos de agua como laguna Tres Palos, donde la Lt fue $13.2 \mathrm{~cm}$ y Pt de $28.6 \mathrm{~g}$ (Román-Contreras, 1979), laguna Mitla donde la Lt fue 11.7 cm (Román-Contreras, 1991), Signoret y Brailovsky (2000) registraron una talla máxima de $13 \mathrm{~cm}$ y el peso varió en un intervalo de 0.20 a 27.4 g, esto para la laguna de Coyuca, Guerrero. En Mexcaltitán, Nayarit, Pérez-Velázquez et al. (2011), reportaron una longitud máxima de $16 \mathrm{~cm}$ y un peso total de 48.8 g., los mismos autores mencionan que se ha observado que estos organismos tienden a ser más grandes en latitudes altas.

Sánchez et al. (2008), observaron diferencias en el crecimiento de machos y hembras de Atya margaritacea A. Milne-Edwards, 1864; en machos fue alométrico positivo y en hembras fue isométrico, contrario a lo observado para el presente estudio donde en ambos sexos se presentó un crecimiento alométrico negativo, lo que indica que los organismos crecen más en talla que en peso (Fig. 8).

El factor de condición (K) fue similar en ambos sexos en todos los meses excepto febrero, en el que las hembras presentaron un índice mayor (Fig. 9). Esto no es igual en todos los crustáceos, Vázquez-López y Ramírez-Pérez (2015) reportaron que los machos de Cardisoma crassum Smith, 1870 presentan una mejor condición que las hembras. En diferentes especies el factor K está influenciado por factores ambientales y biológicos como la cantidad de alimento, temperatura y época reproductiva entre otros (Le Cren, 1951; Franco-López et al., 2010; Cifuentes et al., 2012).

En el presente estudio el $64.6 \%$ del total de los organismos fueron hembras; En todas las recolectas las hembras representaron más del $50 \%$ de la población (Tabla 2, Fig. 10), esto es similar a lo reportado por Román-Contreras (1991) para la laguna de Coyuca donde el número de hembras (53.4\%) fue mayor a lo largo del año; Signoret y Brailovsky (2000) reportaron para el mismo cuerpo de agua que $63.2 \%$ de la población fueron hembras.

\section{Estructura de tallas}

En agosto la precipitación fue menor en comparación con los meses anteriores, debido a ello, algunos estanques perdieron volumen de agua, lo que incrementó su salinidad y la temperatura. El valor medio de la talla en la población fue de $1.78 \mathrm{~cm}$. Se capturaron 166 postlarvas, y 500 organismos en etapa de desarrollo larval, esto gracias a que en el área de estudio las lluvias comenzaron a finales de mayo (2013), por lo que en agosto la población de adultos posiblemente ya había migrado a los ríos.

En octubre la temperatura y la precipitación disminuyeron y la salinidad aumento, se colectaron 78 organismos en estadio postlarval, nueve juveniles y dos adultos (Fig. 11). El valor medio de la talla en la población fue $2.09 \mathrm{~cm}$ de Lt. Román-Contreras (1991) menciona que en octubre (de 1987) la población de M. tenellum estuvo compuesta en su mayoría de organismos 
adultos, lo que posiblemente se debió a que la temporada reproductiva estaba ocurriendo.

En febrero se colectaron 12 organismos, cinco postlarvales, seis juveniles y un organismo maduro. El valor medio de la talla en la población fue de $3.01 \mathrm{~cm}$ de Lt. La disminución en la temperatura y la falta de lluvias en este mes puede ser la razón de la ausencia de organismos.

En mayo se encontraron etapas reproductivas, en este mes se capturaron 89 organismos entre adultos y maduros aun cuando las lluvias se presentaron de manera escaza y la salinidad se mantuvo entre 5 y 7 UPS, en este mes se observaron todos los estadios de M. tenellum (desde postlarvas a organismos maduros). Espinosa-Chaurand et al. (2011), mencionan que los organismos de etapa postlarval $(1.2-2.5 \mathrm{~cm})$ en adelante, requieren menos de $20 \mathrm{UPS}$, lo que concuerda con lo observado en el presente estudio, ya que se capturaron 48 postlarvas y 109 juveniles además de organismos en etapa reproductiva. El valor medio de la talla en la población fue de $3.43 \mathrm{~cm}$ de Lt.

En julio se capturaron 120 organismos, 16 postlarvales, 53 juveniles, 39 adultos y 12 adultos maduros. Las tallas más grandes se reportaron en este mes, el valor medio de la talla en la población fue $3.58 \mathrm{~cm}$ de Lt. observándose un menor número de organismos adultos y maduros, que en mayo; probablemente los organismos de estas tallas ya se encontraban migrando río arriba debido a las lluvias que habían iniciado en 2014. Vega-Villasante et al. (2011b), mencionan que los langostinos de las zonas costeras regresan rio arriba a ríos y lagos y que deben viajar aprovechando las corrientes para reclutarse y migrar. Mcdowall (2007) menciona que la anfidromia es la característica de realizar migraciones en las que diferentes estadios se desplazan y completan partes de una ruta migratoria. Bauer (2013) explica que la mayoría de las especies pertenecientes al género Macrobrachium muestran un patrón de desarrollo anfidromo. Bauer (2011b) menciona que las distancias recorridas por los langostinos pueden ser de kilómetros. En El Salado la arribada de los organismos adultos al estero podría verse retrasada debido a que el área se encuentra rodeada por estructuras artificiales (un aeropuerto, un centro de convenciones, un centro universitario y parte de la zona habitacional de Puerto Vallarta). Espinosa y Rodríguez (1986) manifiestan que los principales impedimentos de las migraciones que provocan la ausencia de Macrobrachium en ríos y cuerpos de agua sobre la franja costera son antrópicos (embalses, represas y contaminación principalmente).

Debido a lo anterior, se puede concluir que: a) la abundancia de M. tenellum varía temporalmente y está relacionada con la temperatura y las precipitaciones, b) en los lugares de captura la salinidad permanece baja, lo que permite la presencia de M. tenellum durante todo el año, c) existen diferencias significativas en la abundancia mensual de M. tenellum en el estero El Salado, d) mayo es el mes de mayor abundancia, e) los machos presentan mayores tallas que las hembras, f) los estadios más abundantes son las postlarvas y juveniles, g) los estadios de adultos y maduros se incrementan en época de lluvias, y h) la proporción sexual en el estero El Salado favorece a las hembras.

\section{AGRADECIMIENTOS}

Los autores agradecen el apoyo del personal del Área Natural Protegida estero El Salado, al director de la misma, Biol. Jaime Alberto Torres Guerrero por las facilidades otorgadas, al Biol. Víctor Enrique Hernández Santos y al Lic. en turismo Julio Cesar Llamas por su valiosa ayuda en los muestreos.

\section{REFERENCIAS}

1. Bauer R., 2011a. Amphidromy and migrations of freshwater shrimps. I. Costs, benefits evolutionary origins and an unusual case of amphidromy. En: C. Fransen, A. Asakura y C.F. Akira 
(Eds.). New Frontiers in Crustacean Biology Proceedings of the TCS Summer Meeting, Tokyo, 20-24 September 2009, 15: 145-156.

2. Bauer R.T., 2011b. Amphidromy and migrations of freshwater shrimps. II. Delivery of hatching larvae to the sea, return juvenile upstream migration, and human impacts. En: C. Fransen, A. Asakura y C.F. Akira (Eds.). New Frontiers in Crustacean Biology Proceedings of the TCS Summer Meeting, Tokyo, 20-24 September 2009, 15: 157-168.

3. Bauer R., 2013. Amphidromy in shrimps: a life cycle between rivers and the sea/Anfidromía en camarones: Un ciclo de vida entre los ríos y el mar. Latin American Journal of Aquatic Research, Studies on Freshwater Decapods in Latín America, 41(4): 633-650.

4. Brusca R.C. y G.J. Brusca, 2003. Invertebrates. Sinauer Associates, Sunderland, Massachusetts.

5. Cifuentes L., M.P. Torres y M. Frías, 1997. El océano y sus recursos II las ciencias del mar: Oceanografía geológica y química. Fondo de Cultura Económica, México.

6. Cifuentes R., J. González, G. Montoya, A. Jara, N. Ortíz, P. Piedra y E. Habit, 2012. Weight-length relationships and condition factor of native fish from San Pedro River, Valdivia River basin, Chile. Gayana 75(2): 101-110

7. Espinosa J.L. y A. Rodríguez, 1986. El Langostino: Un alimento en peligro. Serie medio ambiente en Coatzacoalcos, Volumen X. Centro de Ecodesarrollo. D.F., México.

8. Espinosa-Chaurand L.D., M.A. Vargas-Ceballos, M. Guzmán-Arroyo, H. Nolasco-Soria, O. Carrillo-Farnés, O. Chong-Carrillo y F. Vega-Villasante, 2011. Biología y cultivo de Macrobrachium tenellum: Estado del arte. Hidrobiológica, 21(2): 98-117.

9. Flores R.R., M.L. Morfín y L.D. Espinosa, 2012. Temperatura óptima y preferencia térmica del camarón de río Macrobrachium tenellum en la costa tropical del Pacífico Mexicano. Boletín del Instituto de Pesca, São Paulo, 38(2): 121-130.

10. Franco-López J., C.B. Sánchez, H.B. Escorcia, L.G .Abarca-Arena, T.C. Ferreira y H. VázquezLópez, 2010. Biological and ecological aspects regarding Cynoscion nothus Holbrook, 1855 (Perciforms: Sciaenidae). Research Journal of Fisheries and Hydrobiology, 5(2): 66-75.

11. García-Guerrero M.U., F. Becerril-Morales, F. Vega-Villasante y L.D. Espinosa-Chaurand, 2013. Los langostinos del género Macrobrachium con importancia económica y pesquera en América Latina: conocimiento actual, rol ecológico y conservación. Latin American Journal of Aquatic Research, 41(4): 651-675.

12. Hendrickx M.E., 1995. Camarones. En: W. Fischer, F. Krupp, W. Scneider, C. Sommer, K.E. Carpenter y V.H. Niem (Eds.). Guía FAO para la identificación de especies para los fines de pesca. Pacífico centro oriental. Vol. 1. Plantas e invertebrados. Roma, Italia.

13. Hernández-Murugan L., G. Ruiz-Campos y A.M. Maeda-Martínez, 2007. Freshwater shrimp of the genus Macrobrachium (Decapoda: Palaemonidae) from the Baja California peninsula, Mexico. Journal of Crustacean Biology, 27(2): 351-369.

14. Hernández-Sandoval P., 2008. Efecto de la temperatura en el crecimiento y supervivencia del 
langostino Macrobrachium occidentale y del acocil Cherax quadricarinatus (Tesis de maestría, Instituto Politécnico Nacional, México).

15. Holthuis L.B., 1952. A general revision of the Palaemonidae (Crustacea, Decapoda, Natantia) of the Americas. II. The subfamily Palaemoninae. Occasional Papers of the Allan Hancock Foundation, 12(1): 54-130.

16. LeCren E.D., 1951. The length-weight relationship and seasonal cycle in gonad weight and condition in the perch (Perca fluviatilis). The Journal of Animal Ecology, 20: 201-212.

17. McDowall R., 2007. On amphidromy, a distinct form of diadromy in aquatic organisms. Fish and Fisheries, 8: 1-13.

18. Murphy N.P. y C.M. Austin, 2005. Phylogenetic relationships of the globally distributed freshwater prawn genus Macrobrachium (Crustacea: Decapoda: Palaemonidae): biogeography, taxonomy and the convergent evolution of abbreviated larval development. Zoologica Scripta, 34(2): 187-197.

19. Olugbenga E.L. y R.O. Taiwo, 2013. Growth patterns, sex ratios and fecundity estimates in blue crab (Callinectes amnicola) from Yewa River, Southwest Nigeria. Advances in Life Science and Technology, 7: 24-34.

20. Pauly D., 1984. Some simple methods for the assessment of tropical fish stocks. FAO Fisheries Technical Paper. No 234.

21. Pérez-Velázquez P.A., S. Hernández-Ventura, P. Ulloa-Ramírez, J.L. Patiño-Valencia y J. TovarÁvila, 2011. La pesca del langostino (Macrobrachium tenellum) en la laguna de Mexcaltitán, Nayarit, una alternativa económica regional. Ciencia Pesquera, 19(1): 14.

22. Ponce-Palafox J.T., F.C. Arana-Magallón, H. Cabanillas-Beltrán, H. Esparza-Leal e I. de Blas, 2002. Bases biológicas y técnicas para el cultivo de los camarones de agua dulce nativos del Pacífico Americano Macrobrachium tenellum (Smith, 1871) y M. americanum (Bate, 1968). Congreso Iberoamericano Virtual de Acuicultura, CIVA. p. 534-546.

23. Ponce-Palafox J.T., G.M. García-Ulloa, J.L. Arredondo-Figueroa, D. Hernández-Ocampo, J. DíazÁlvarez, G. Aldama-Rojas, y H. Esparza-Leal, 2006. El cultivo del camarón de agua dulce Macrobrachium tenellum en estanques rústicos. En: Comunicación Científica. IV Congreso Iberoamericano Virtual de Acuicultura. p. 655-660.

24. Ricker W.E., 1975. Computation and interpretation of biological statistics of fish populations. Bulletin of the Fisheries Research Board of Canada. Bulletin 191.

25. Román-Contreras R., 1979. Contribución al conocimiento de la biología y ecología de Macrobrachium tenellum (Smith ,1871) (Crustacea, Decapoda, Palaemonidae). Anales del Instituto de Ciencias del Mar y Limnología, 6(2): 137-160.

26. Román-Contreras R., 1991. Ecología de Macrobrachium tenellum (Decapoda: Palaemonidae) en la laguna de Coyuca, Pacífico de México. Anales del Instituto de Ciencias del Mar y Limnología, 18(1): 109-160. 
27. Sánchez J.P., R.A. Beltrán y J.P.L. Ramírez, 2008. Crecimiento y reproducción del camarón Atya margaritacea (Decapoda: Atyidae) en el río Presidio, Sinaloa, México. Revista de Biología Tropical, 56(2): 513-522.

28. SCT SENEAM (Secretaría de Comunicaciones y Transportes, Servicios a la Navegación en el Espacio Aéreo Mexicano, 2015. Tabla climatológica de aeródromo. Tablas de precipitación diaria del Aeropuerto de Puerto Vallarta del 2013, 2014 y 2016, Febrero 11, 2016.

29. Signoret G. y D. Brailovsky, 2002. Population study of Macrobrachium tenellum (smith 1871) en Coyuca de Benítez, Guerrero, México. En: E. Escobar y F. Alvarez (Eds.) Modern Approaches to the Study of Crustacean. Springer USA.

30. Vázquez-López H. y T. Ramírez-Pérez, 2015. Aspects of growth in the terrestrial crab Cardisoma crassum Smith, 1870 (Crustacea: Brachyura: Gecarcinidae) from El Salado Estuary, Puerto Vallarta, Jalisco, México. Mitteilungen Klosterneuburg 65 (2): 82-99.

31. Vega-Villasante F., J.D. Galaviz-Parada, M. Guzmán-Arroyo, C.A. Flores-Zepeda y L.D. Espinosa-Chaurand, 2011.a Efecto de diferentes salinidades sobre el crecimiento y supervivencia de juveniles del langostino de río Macrobrachium tenellum Smith, 1871. Zootecnia Tropical, 29(4): 467473.

32. Vega-Villasante F, L.D.C. Espinosa, S.G. Yamasaki, E.J. Cortés, M.U.G. García, A.L.M. Cupul, H.S. Nolasco y M.A. Guzmán, 2011b. Acuicultura del langostino Macrobrachium tenellum. Engorda en estanques semirrústicos. Universidad de Guadalajara, Jalisco, México. México.

33. Villalobos-Hiriart J.L., A. Cantú y E. Lira-Fernández, 1993. Los crustáceos de agua dulce de México. Revista de la Sociedad Mexicana de Historia Natural, 44: 267-290.

34. Zar J.H., 2009. Biostatistical Analysis. Pearson Prentice Hall, Northern Illinois University. 